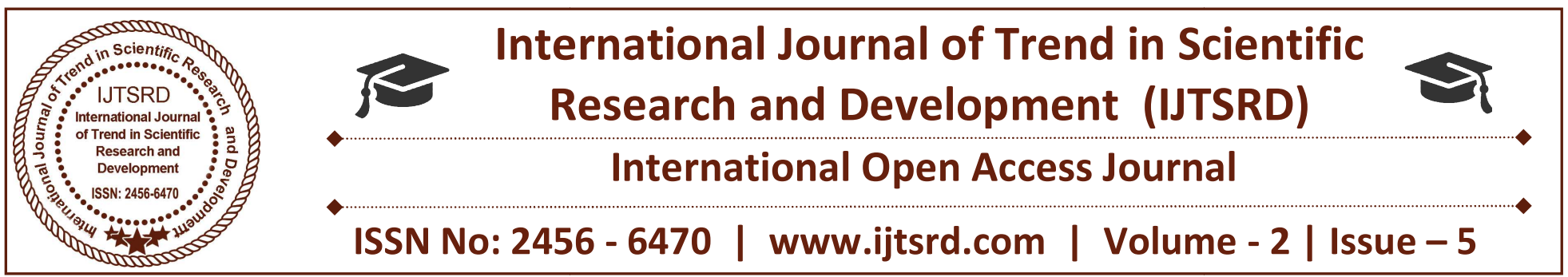

\title{
Application of Adaptive Filter in Neural Network
}

\author{
S. Sree Ragavi, S. Ramadevi \\ Department of Mathematics, Vivekanandha College of Arts and Sciences for Women \\ (Autonomous), Elayampalayam, Thiruchengode, Namakkal, Tamil Nadu, India
}

\section{ABSTRACT}

The snag of common linear filtered- $\epsilon$ Adaptive Inverse Control (AIC) method is modify to hold with the characteristic of non-linear object with time delay and corresponding filtered $\in$-adaptive Algorithm based on Real-Time Recurrent Learning (RTRL) is presented to identify the parameters and design the controller. The simulation result on a non-linear ship model of "The R.O.V. Zeefakkel" and Adaptive PID control keeps the same dynamic response performance, and also mathematical model for ship Course keeping be discussed. A neural network adaptive filter is introduced for the removal of impulse noise in digital images.

Keyword: Filtered-E Adaptive Inverse Control, Ship course keeping, impulse noise detection and removal.

\section{INTRODUCTION}

Adaptive Inverse Control (AIC) is novel approaches which can make a plant track the input command signal with a controller whose transfer function approximates the inverse of plant transfer function. Compared with traditional methods, AIC can achieve specified dynamic responses more easily and has better ability of disturbance rejection. Simulation comparison with previous scheme and adaptive PID control are performed on the non-linear ship maneuvering to test the effect of improved algorithm. The impulse noise detection and removal of impulse noise while preserving the integrity of an image expressed mathematically be discussed.

\section{The Improved Filtered- $\in$ Adaptive Inverse Control}

The Single Input Single Output (SISO) discrete-time nonlinear system to be considered is described by $n$ dimension state equations as below

$$
\left\{\begin{array}{c}
x(k+1)=f[x(k), u(k)], f(0,0)=0 \\
y(k)=h[x(k)]
\end{array}\right.
$$

Where $f($.$) is a non-linear function, u(k)$ and $y(k)$ respect output and input variables, respectively. The principles of inverse control can be extended to deal with non-linear system, through non-linear system has no strict inverse model. Whereas a linear system possesses a unique inverse, non-linear systems have only local inverses, valid in a bounded region of the signal space. The non-linear system is supposed to has Bounded-Input Bounded Output(BIBO) stability, i.e., there are existing constants $C_{1}$ and $C_{2}$, and for the input $u(k) \in s_{u}, s_{u}=\left\{u:|u(k)| \leq C_{1}, \forall k \geq k_{0}\right\}$, the system output $y(k)$ satisfies: $|\mathrm{y}(\mathrm{k})| \leq C_{2}$. If its linearized system is observable at balance point $x=0$, the non-linear system described by (1) has local NARMA (Non-linear Auto-Regressive Average) model as follows

$$
\begin{gathered}
y(k+d)=F[y(k), \ldots \\
\left.y\left(k-n_{y}\right), u(k), \ldots, u\left(k-n_{u}\right)\right]
\end{gathered}
$$

Where $n_{u}$ and $n_{y}$ are the orders of input time series and output time series respectively: $n_{u}<n_{y}, d$ is system delay which can ensure the existence of system inverse model, and $F($.$) is certain non-linear$ function. If $F($.$) is monotonic change with (k)$, system (1)is invertible in the input spectrum $S_{u}$. If the input signal is $y_{m}(k+1)$ at $\operatorname{time}(k+1)$, the controller can be expressed as

$u(k)=g\left[y_{m}(k+1), y(k), \ldots \ldots, y\left(k-n_{y}+\right.\right.$ $1, u k-1, \ldots \ldots \ldots k-n u+1(3)$

Function $F($.$) and g($.$) are both unique. Under the$ dreive of $u(k)$, the plant output will track the input signal of the inverse controller. 


\section{Identification of Delay Time Complexity}

The output of a non-linear system with time-lag characteristic can be described as below form

$$
\begin{gathered}
y(k)=f(x(k-d), \ldots, x(k-m-d), \\
y(k-1), \ldots \ldots y(k-n))
\end{gathered}
$$

Where $x(k)$ and $y(k)$ represent the input and output of system at time $k, m$ and $n$ are the input and output order, $d$ is system delay time, and $f($.$) is the transfer$ function of this non-linear system.

If we use neural network to model such a non-linear plant, the training effectiveness will be greatly influenced by the samples of input vector collected in different range. Therefore, such network can be used as delay-time identifier. Here a non-linear autoregressive with exogenous inputs (NARX) network is used, which can be described as

$$
\begin{gathered}
x(k)=[x(k), \ldots, x(k-a), \\
y(k-1), \ldots y(k-b)]
\end{gathered}
$$

Where $\mathrm{a}$ and $\mathrm{b}$ denotes the length of tapped delay lines of input and output feedback, respectively. The plant input/output data pairs collected in certain time period are used to train the NARX network, and many research result have demonstrated that the sample range of $u(k)$ changing from not including the first delayed input signal $a=d-1$ to including it, i.e., $a=d-1 \rightarrow a=d$, will cause the training effectiveness having a great improvement, and hence the delay time $\mathrm{d}$ of controlled plant can be confirmed.

\section{Improved Filtered- $\varepsilon$ Algorithm}

The NARX filter is also used to the controller modeling. For including self-feedback, it must be adapted using a method such as Real-Time Recurrent Learning (RTRL) or Back-propogation Through Time (BPTT). Considering the real-time requirement of practical application, we propose the filtered- $\varepsilon$ algorithm based on RTRL. To model $\hat{C}$, RTRL usually needs to obtain the error $\varepsilon^{*}$ between $\hat{C}$ and the ideal controller outputs, and then $\hat{C}$ parameters can be adjusted via the principle of gradient descent to minimize the objective function $J_{C}=\left(\varepsilon^{*}\right)^{2} / 2$, but $\varepsilon^{*}$ is unknown in practical AIC implementation. In improved filtered- $\varepsilon$ algorithm, $\varepsilon^{*}$ is replaced by system error $\varepsilon^{\prime}$ which is filtered by $\hat{P}_{\Delta}^{-1}$ to adapt $\hat{C}$ parameters. The update algorithm can be expressed as follows

$$
\begin{array}{r}
W_{C}(k+1)=W_{C}(k) \\
+\mu(1-\eta)\left(-\nabla_{c}(k)\right)+\eta W_{c}(k)
\end{array}
$$

Where $\mu$ and $\eta$ denote the step size and momentum co-efficient respectively, and $\nabla_{c}(k)$ represents the gradient of $J_{C}$ with respect to controller weights $W_{c}$ :

$$
\begin{array}{r}
\nabla_{c}(k)=\frac{d J_{C}}{d W_{c}}=\frac{1}{2} \frac{d \varepsilon^{\prime}(k)^{2}}{d W_{c}} \\
=\varepsilon^{\prime}(k) \frac{d(\widehat{u}(k)-\widehat{u}(k-\Delta)}{d W_{c}} \\
=-\varepsilon^{\prime}(k) \frac{d \widehat{u}(k-\Delta)}{d W_{c}}
\end{array}
$$

Where $\frac{d \hat{u}(k-\Delta)}{d W_{c}}$ is the $\Delta$-delayed partial derivative of $\hat{C}$ output $\hat{u}(k)$ with respect to its weights $w_{c}$ at time $k$. It is updated by the chain rule:

$$
\begin{aligned}
& \frac{d \hat{u}(k)}{d W_{c}} \\
& =\frac{\partial \hat{u}(k)}{\partial W_{c}}+\sum_{n=0}^{a} \frac{\partial \hat{u}(k)}{\partial r(k-n)} \frac{\partial r(k-n)}{\partial W_{c}} \\
& \sum_{m=1}^{b} \frac{\partial \hat{u}(k)}{\partial \hat{u}(k-m)} \frac{\partial \hat{u}(k-m)}{\partial W_{c}}
\end{aligned}
$$

$$
=\frac{\partial \widehat{u}(k)}{\partial W_{c}}+\sum_{m=1}^{b} \frac{\partial \widehat{u}(k)}{\partial \widehat{u}(k-m)} \frac{\partial \widehat{u}(k-m)}{\partial W_{c}}
$$

The terms $\frac{\partial \widehat{u}(k)}{\partial W_{c}}, \frac{\partial \widehat{u}(k)}{\partial r(k-n)}$ and $\frac{\partial \widehat{u}(k)}{\partial \widehat{u}(k-m)}$ are the direct effect of a change in the weights, previous inputs and outputs on the current output respectively, and these partial derivatives may be computed directly using the back-propagation algorithm. $\frac{\partial \widehat{u}(k-m)}{\partial W_{c}}$ is simply a previously-calculated and stored value of $\frac{\partial \hat{u}(k)}{\partial W_{c}}$. For i $=0,-1,-2, \ldots, \frac{\partial \hat{u}(i)}{\partial W_{c}}$ are set to zero. We also note that $\frac{\partial r(k-n)}{\partial W_{c}}$, the partial derivative of the input of $\hat{C}$ with respect to its weights, is zero for allk.

\section{SIMULATION RESEARCH}

We apply the improved Filtered- $\varepsilon$ AIC method proposed in this paper to the control of non-linear ship maneuvering. As a typical non-linear system with time-lag characteristic, ship can be described by the non-linear model.

$$
T \ddot{\psi}+\dot{\psi}+\alpha \dot{\psi}^{3}=K \delta+\omega
$$

Where $\psi$ is the yaw angle, $\delta$ is the control rudder angle, $T, K$ and $\alpha$ are the model parameter, and $\omega$ is the external disturbance.

The ship model used in the simulation study is adapted from Van Amerongen (1982). In this 
reference , "The R.O.V. Zeefakkel" is described by the following set of parameters and the ship 'R.O.V. Zeefakkel' of $45 \mathrm{~m}$ length can be represented by the non-linear mathematical model and the model parameters for a ship speed of 10 knots be Ship length $L=45 \mathrm{~m}$, service speed $v=5.0 \mathrm{~m} / \mathrm{s}$, gain constant $k=0.5 s^{-1}$, time constant $T=31 \mathrm{~s}$, and Norrbin coefficients $n_{1}=1.0, n_{3}=0.4(s)^{2}$. In order to evaluate the performance of the improved filtered- $\varepsilon$-AIC scheme, conventional filtered- $\varepsilon$ AIC and adaptive PID control are also performed. In the simulation, the sampling period

$$
T^{\prime}=0.1 s
$$

(a)Firstly the ship $\pm 10^{\circ}$ course-changing test is performed without external disturbance, namely $\omega=0$. At $k=300 s$, the ship time constant constant $T$ changes from $31 \mathrm{~s}$ to $45 \mathrm{~s}$. Clearly the conditional filtered- $\varepsilon$ AIC has much better dynamic performances than that of the adaptive PID control, but there existing static error in it for the influence of the change of plant delay time. In contrast, the improved scheme avoids static error and at the mean time, it has kept the same dynamic performances as conventional filtered- $\varepsilon$ AIC method.

(a) The second simulation to be examined is the disturbance rejection ability. The external disturbance is chosen as

$$
\omega=4.5^{\circ} \mathrm{H}_{1}+3.44^{\circ} \mathrm{H}_{2}
$$

Where $H_{1}$ and $H_{2}$ are normally distributed random numbers with mean 0 , varience $\sigma^{2}=1$. The comparison of the response curves between improved method and conventional ones. It can be clearly seen that the improved scheme has better disturbance rejection ability.

\section{Ship Course-Keeping Mathematical Model}

Equation (1) gives linear first-Order Nomoto model which is used for ship course-keeping

$$
\ddot{\psi}+\frac{1}{T_{0}} \dot{\psi}=\frac{K_{0}}{T_{0}} \delta,
$$

Among these, $\psi$ is the ship heading angle, $\delta$ is the rudder angle, $K_{0}$ is the steering gain index, $T_{0}$ is the ship following index. It presents a Nomoto model based responding type of non-linear mathematical model, that is using a non-linear item $\left(K_{0} / T_{0}\right) H(\dot{\psi})$ to replace $\dot{\psi} / T_{0}$, and

$$
H(\dot{\psi})=\dot{\psi}+\beta \dot{\psi}^{3},
$$

Where $\alpha, \beta$ represent the scale co-efficient of the first and the third power of the rate of turning $\dot{\psi}$. Specific parameter value depends on the factors like ship type, stowage, ship speed, etc. Taking $\psi$ as ship heading angle, then $\dot{\psi}=r$; meanwhile, taking the frequently influence from uncertain interference such as wind, wave and current into consideration, the non-linear dynamic equation of ship course-keeping system could be written as:

$$
\left\{\begin{array}{c}
\dot{\psi}=r \\
\dot{r}=-\frac{K_{0}}{T_{0}}\left(\alpha r+\beta r^{3}\right)+\frac{K_{0}}{T_{0}} \delta+\Delta,
\end{array}\right.
$$

Where $\Delta$ is the uncertain interference. According to engineering practice, $\Delta$ is usually bounded disturbance, assuming there is an unknown constant could meet the equation (1) on the basis of hypothesis.

$\|\Delta\|_{\infty} \leq \rho, \rho$ is a unknown positive constant. (4)

\section{Impulse Noise Detection and Removal}

When an image is coded and transmitted over a noisy channel or degraded by electrical sensor noise, as in a vidiction TV camera, degradation appear as "salt and-paper" noise (i.e., positive and negative impulses). Two models have been proposed to describe impulsive noise (Justusson, 1981). The first model assumes fixed values for all the impulses, and the second model allows the impulses to follow a random distribution. In this work, only the first model is dealt with. The model is mathematically expressed as

$$
x_{i}=\left\{\begin{array}{c}
d_{p} \text { with probability } P_{p} \\
d_{n} \text { with probability } P_{n} \\
f_{i} \text { with probability } 1-\left(P_{p}+P_{n}\right)
\end{array}\right.
$$

Where $f_{i}$ denotes the uncorruped pixel values, $d_{p}$ denotes the fixed value of the positive impulses, $d_{n}$ denotes the fixed value of the negative impulses, and $x_{i}$ denotes the pixel values of the degraded images.

Noisy pixels can be characterized by their local statistical properties. To extract features from local statistics, a window is used to pass through the entire noise degraded image. Theoretically, the size of the window is arbitrary. However, a $3 \times 3$ window is good enough in most applications. From the small window, useful local features are obtained, such as mean, median, variance, range, and extreme range. Noise detection is then treated as a pattern classification problem. Assume that $M$ features are measured from 
each input pattern. Each set of $M$ features is considered as a vector in the M-dimensional feature space. Pattern classification is accomplished by a selforganising neural network which is capable of cluster analysis for image data.

The neural network proposed in this paper is a simplified version of ART I (Carpenter \& Gross berg, 1987). However, it is capable of grey - level classification. It has two layers: the input layer and the output layer. These layers are connected by feed forward paths. The nodes of output layer are lateral inhibitive to one another. They are designed as a competitive network capable of choosing the winning node. The set of weights $u_{i j}$ for any specific node $i$ constitute the prototype pattern for clusteri. The clustering algorithm starts from isolated patterns and coalesces the nearest patterns or groups according to a threshold from the bottom up to form hierarchies. The essential point of this algorithm is to build up the clusters using the Euclidean distance measure between the input $s$ and the weights $u_{i}$. Assume a sequence of pattern samples

$$
s=\left(s_{1}, s_{2}, \ldots s_{M}\right) \in R^{M}
$$

and a set of weight vectors

$$
u_{i}=\left(u_{i 1}, u_{i 2}, \ldots, u_{i M}\right) \in R^{M}
$$

It is well known that similarities exist between ART and Kohonen's self-organising map.

\section{CONCLUSION}

In this paper, an improved nonlinear filtered- $\varepsilon$ method is introduced and adaptive PID control are performed on the control of non-linear ship maneuvering and also mathematical model for ship Course-Keeping and impulsive noise be expressed.

\section{REFERENCE}

1. Widrow B, Walach E. Adaptive inverse control. Englewood cliffs, N J: Prentice-Hall press, 1996.

2. Zhang Y H, Liu S X. An adaptive automatic ship steering system. Shipbuilding of china, 1991.

3. Nieminen, A., Heinonen, P., \& Neuvo, Y. (1987). A new class of detail-preserving filters for image processing. IEEE Transactions pattern Analysis Machine Intelligence.

4. Zhang Xianku, Lu Xiaofei, Guo Chen, Yang Yansheng, Robust Neural Network Control for Ship's Course-Keeping, Journal of Ship Mechanics, Vol. 10, Issue 5, 2006.

5. Du Gaang, Zhan Xingqun, Zhong Shan, Zhang Weiming, Adaptive inverse control of non-linear ship manueuvering based on Neural Networks, Measuring and Testing Technique, Vol. 24 Issue 4, 2005.

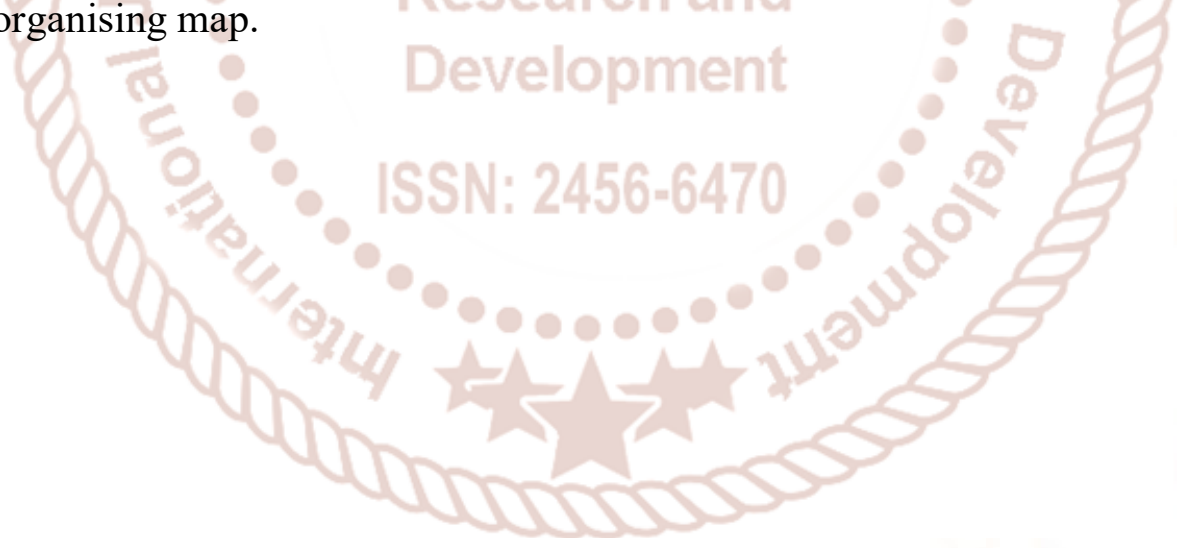

